\title{
DETECTOR DEVELOPMENT AND CALIBRATION
}

\author{
PERFORMANCE OF A NEUTRON POLARIMETER TO \\ MEASURE THE ELECTRIC FORM FACTOR OF THE NEUTRON
}

T. Eden, R. Madey, W.M. Zhang, B.D. Anderson, A.R. Baldwin,

J. Schambach, E. Steinfelds and J.W. Watson

Center for Nuclear Research, Kent State University, Kent, Ohio 44242

S. Kowalski

Massachusetts Institute of Technology, Cambridge, Massachusetts 02139

\author{
J.J. Kelly \\ University of Maryland, College Park, Maryland 20742 \\ J.M. Finn, P. Markowitz \\ College of William and Mary, Williamsburg, Virginia 23185 \\ R. Lourie \\ University of Virginia, Charlottesville, Virginia 22904 \\ P.J. Pella \\ Gettysburg College, Gettysburg, Pennsylvania 17325 \\ J. Cameron, C.C. Foster, B. Ni, M. Spraker, and E.J. Stephenson \\ Indiana University Cyclotron Facility, Bloomington, Indiana 47405
}

In proposals to the Bates electron accelerator facility and to the Continuous Electron Beam Accelerator Facility (CEBAF), we proposed to determine the electric form factor $G_{E}^{n}$ of the neutron by scattering longitudinally-polarized electrons quasielastically from deuterium and measuring the transverse polarization component $p_{s^{\prime}}$ of the recoil neutron. In the impulse approximation, this transverse neutron polarization component $p_{s^{\prime}}$, which lies in the scattering plane but normal to the neutron momentum, is directly proportional to $G_{E}^{n} \cdot{ }^{1}$ For the kinematic conditions of the experiment at Bates, the kinetic energy of the neutron is centered at $136 \mathrm{MeV}$. The kinetic energies of the neutrons in the CEBAF experiment range from $80 \mathrm{MeV}$ to $1 \mathrm{GeV}$.

The principle of the measurement of the neutron polarization component $p_{s^{\prime}}$ is based on the fact that there is an asymmetry in the scattering of polarized neutrons at an angle $\theta$ from unpolarized hydrogen nuclei. The up-down asymmetry $\xi(\theta, \phi)$ is

$$
\frac{\left(N_{U}-N_{D}\right)}{\left(N_{U}+N_{D}\right)} \equiv \xi(\theta, \phi)=\vec{p}_{s^{\prime}} \cdot \vec{A}_{y}(\theta)=p_{s^{\prime}} A_{y}(\theta) \cos \phi,
$$

where $N_{U}\left(N_{D}\right)$ is the number of neutrons scattered upward(downward), $\vec{A}_{y}(\theta)$ is the analyzing power of n-p scattering at the polar angle $\theta$ (the angle between the $\mathrm{z}$-axis and 
the momentum vector of the scattered neutron), and the azimuthal angle $\phi$ (the angle between the vertical $y-z$ plane of the polarimeter and the secondary scattering plane). The asymmetry averaged over the angular acceptances of the polarimeter is

$$
\bar{\xi}=p_{s^{\prime}} \overline{A_{y}}=p_{s^{\prime}}<A_{y}(\theta) \cos \phi>
$$

Here $\overline{A_{y}}$ is the analyzing power averaged over the $\theta$ and $\phi$ acceptances of the polarimeter. According to Eq. (2), the neutron polarization can be measured with a polarimeter of known average analyzing power $\overline{A_{y}}$ by measuring the average scattering asymmetry $\bar{\xi}$ of a neutron flux of known polarization $p_{s^{\prime}}$. The purpose of IUCF E326 was to measure $\overline{A_{y}}$ for polarized neutrons with a kinetic energy of about $135 \mathrm{MeV}$.

To obtain a neutron flux of known polarization, we used the $0^{+} \rightarrow 0^{+}$transition to the isobaric analog state (IAS) in the ${ }^{14} C(\vec{p}, \vec{n}){ }^{14} N(2.31 \mathrm{MeV})$ reaction at zero degrees. The $Q$-value is $-0.63 \mathrm{MeV}$ to the ground state and $-2.94 \mathrm{MeV}$ to the $2.31 \mathrm{MeV}$ state. If the incident protons are polarized normal to the reaction plane, and if the interaction conserves angular momentum and parity, the general relationship ${ }^{2,3}$ between the neutron polarization $p_{n}$ and the proton polarization $p_{p}$ is

$$
p_{n}\left[1+p_{p} A_{y}(\theta)\right]=P(\theta)+p_{p} D_{N N^{\prime}}
$$

where $A_{y}(\theta)$ is the analyzing power of the reaction, $P(\theta)$ is the polarization function of the reaction, and $D_{N N^{\prime}}$ is the polarization-transfer coefficient. A $(\vec{p}, \vec{n})$ reaction on a $J^{\pi}=0^{+}$ target to a $J^{\pi}=0^{+}$state in the residual nucleus is a pure nonspin-flip transition. Because the proton transfers its spin to the neutron without flipping the spin, the polarization transfer coefficient $D_{N N^{\prime}} \equiv 1$ for a $0^{+} \rightarrow 0^{+}$transition. At $0^{\circ}$, the analyzing power $A_{y}\left(0^{\circ}\right)$ and the polarization function $P\left(0^{\circ}\right)$ vanish identically; hence, for a $0^{+} \rightarrow 0^{+}$transition at $0^{\circ}$, Eq. (3) simplifies to $p_{n}=p_{p}$. Of the multitude of known $0^{+} \rightarrow 0^{+}$transitions, the ${ }^{14} C(\vec{p}, \vec{n}){ }^{14} N(2.31 \mathrm{MeV})$ is desirable because the cross section at $0^{\circ}$ is large and the 2.31 $\mathrm{MeV}$ state can be separated easily from neighboring states.

\section{Experimental Procedure}

These measurements (E326) were performed with polarized protons at the Indiana University Cyclotron Facility (IUCF). The detector station that housed the polarimeter was located outside of the beam swinger facility on the $0^{\circ}$ line at a flight path of $65.33 \mathrm{~m}$ from the target to the midpoint of the four front analyzing detectors in the polarimeter. A polarimeter located in the beam line (BL-2) between the two cyclotrons is used to determine the beam polarization by scattering the protons from a ${ }^{4} \mathrm{He}$ target. Scattered protons are detected by a pair of detectors mounted symmetrically to the left and right of the beam. The difference in the left and right yields are compared with the known analyzing power of the $\vec{p}-{ }^{4} \mathrm{He}$ elastic scattering reaction at a given energy. The beam polarization was measured periodically with data acquisition and analysis software supplied by the IUCF. During a beam polarization run, the extracted scattering yields were used to determine the beam polarization and its associated uncertainty. The proton beam polarization averaged over the duration of these measurements was $0.725 \pm 0.001 \pm 0.015$. The uncertainty of \pm 0.001 is statistical only. Other errors in the system arise from the calibration of the BL-2 
polarimeter, the quality of the background subtraction in the BL-2 polarimeter, and the transport of the proton beam through the cyclotron. The uncertainty in the analyzing power of the BL-2 polarimeter is typically $0.5 \%$. Background subtraction introduces an uncertainty at a level of 1 to $2 \%$. The uncertainty in the proton beam polarization from these factors is estimated to be about \pm 0.015 , about an order of magnitude larger than the statistical uncertainty.

Because the inside dimensions of the detector stations at the IUCF are not large enough to accomodate vertical mounting of the rear detectors of the neutron polarimeter, the detectors were mounted horizontally, which is the same orientation required for measurements of $G_{E}^{n}$. The horizontal orientation of the neutron polarimeter requires a sideways polarized beam for calibration. A superconducting solenoid upstream of the target was used to provide sideways polarized protons at the target, which is located just upstream of the dump magnet in the beam swinger facility. Because the polarization of the neutrons is perpendicular to the magnetic field in the dump magnet, the neutrons precess as they traverse the dump magnet. The precession $\Delta \theta_{s}$ of the neutron spin can be determined from the following expression:

$$
\Delta \theta_{s}=\gamma \frac{g_{n}}{2} \Delta \theta_{p}
$$

Here $g_{n}$ is the intrinsic spin $g$-factor for the neutron, $\gamma$ is the Lorentz factor for the neutron, and $\Delta \theta_{p}$ is the proton bend angle in the dump magnet, which is $12.5^{\circ}$. Equation (4) is a reliable measure of $\Delta \theta_{s}$ provided that the following integrations are equal for the neutrons and the protons; that is,

$$
\int B d l_{p}=\int B d l_{n}
$$

where $d l_{p}$ and $d l_{n}$ are the differential path lengths for the proton and the neutron, respectively, and $B$ is the magnetic field strength that depends on the position of the particle as it traverses the magnet. Because of the relatively small bend angle $\Delta \theta_{p}$ and because the beam dump is at a relatively large distance of about 22 feet from the entrance to the dump magnet, the difference in these two integrals is expected to be small. With $\left|g_{n} / 2\right|=-1.91$ and $\Delta \theta_{p}=12.5^{\circ}$, we find $\Delta \theta_{s}=27.3^{\circ}$ for $T_{p}=140 \mathrm{MeV}(\gamma=1.14)$ in the counterclockwise direction; however, because the protons follow a curved path and pass through more of the fringe field of the dump magnet than the neutrons, $\int B d l_{n}$ is slightly smaller than $\int B d l_{p}$. We estimate $\Delta \theta_{s}=25^{\circ} \pm 2^{\circ} ;$ accordingly, the sideways component of the neutron polarization is reduced by about $10 \%$ :

$$
p_{n}=p_{p} \cos \left(25^{\circ} \pm 2^{\circ}\right)=(0.906 \pm 0.015) p_{p} .
$$

For $p_{p}=0.725 \pm 0.015, p_{n}=0.66 \pm 0.02$.

\section{Performance of the Polarimeter}

Neutron excitation energy spectra from the ${ }^{14} C(\vec{p}, \vec{n})^{14} N$ reaction are shown in Figs. 1 and 2. The fact that the $0^{+}$state at $2.31 \mathrm{MeV}$ of excitation energy is separated clearly from the $1^{+}$state at $3.95 \mathrm{MeV}$ of excitation energy allows the yield under the $0^{+}$calibration peak to be extracted with a small uncertainty. 
Figure 1. Spectrum of scattered neutrons with sideways polarization of the incident neutrons to the right. In panel (a) the neutrons are scattered to the top detectors; and in panel (b) the neutrons are scattered to the bottom detectors.
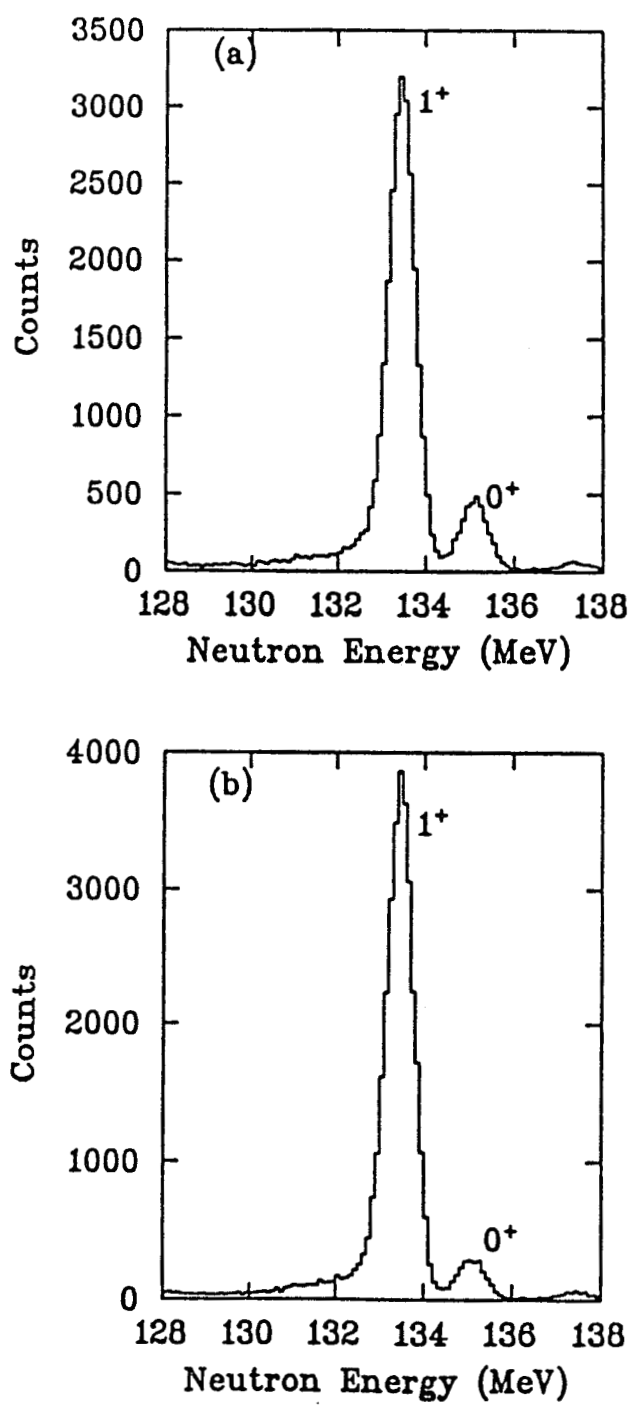

The first objective for evaluating the performance of the polarimeter was the desire for a large "instrumental figure of merit", given by the relationship:

$$
\eta^{*}=\left(\overline{A_{y}}\right)^{2} \epsilon,
$$

where the efficiency $\epsilon$ of the neutron polarimeter is the probability that a neutron entering the polarimeter will produce an event that satisfies all thresholds and cuts. Events are rejected when the velocity of the scattered neutron $v_{s c}$ is not sufficiently close to the velocity $v_{n p}$ calculated for elastic $\mathrm{n}-\mathrm{p}$ scattering. The value of $\eta^{*}$ depends on the cut on the velocity ratio $R_{v}\left(\equiv v_{s c} / v_{n p}\right)$. We examine $\eta^{*}$ as a function of the software $R_{v}$ cut. A second objective is to make $\overline{A_{y}}$ large in order to minimize sensitivity to instrumental asymmetries in the polarimeter; therefore, our goal is to seek a large value of $\overline{A_{y}}$ while maximizing $\eta^{*}$.

In Fig. 3, we present a spectrum of the velocity ratio $R_{v}$ for $T_{n}=133.8 \mathrm{MeV}$; these 


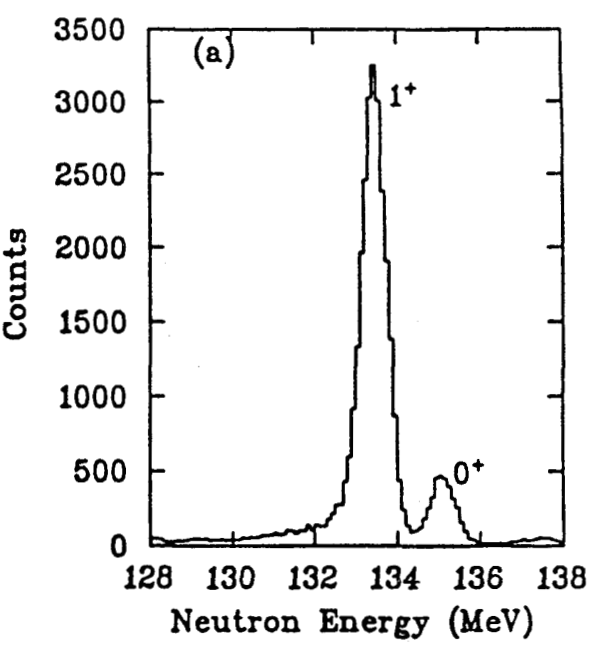

Figure 2. Spectrum of scattered neutrons with sideways polarization of the incident neutrons to the left. In panel (a) the neutrons are scattered to the bottom detectors; and in panel (b) the neutrons are scattered to the top detectors.

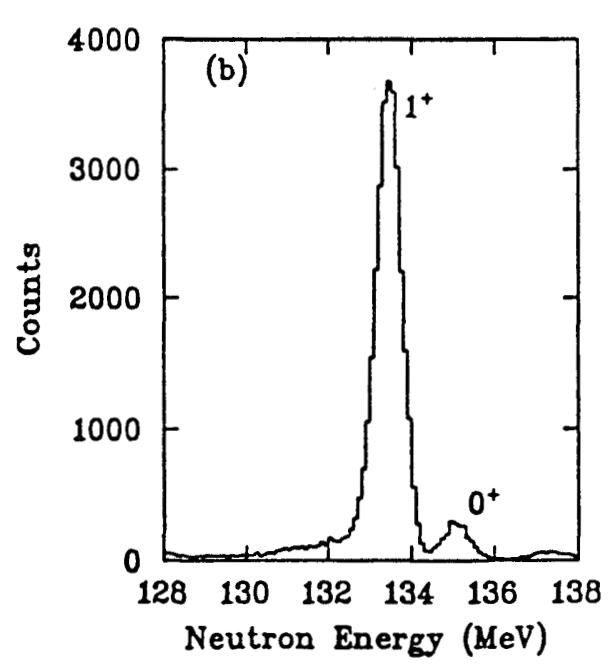

data are from the strong ${ }^{14} N(3.95 \mathrm{MeV})$ state excited in the ${ }^{14} C(\vec{p}, \vec{n}){ }^{14} N$ reaction. The $R_{v}$ spectrum has a distinct, nearly Gaussian, peak centered at $R_{v}=1$ from n-p elastic scattering events, and a continuum of events from reactions on carbon with a peak at $R_{v}=0.87$. The results of five $R_{v}$ cuts that were analyzed for the run without the collimator (discussed below) are shown in Table I.

With a mean flight path of $2.0 \mathrm{~m}$ between the midpoint of the front scatterers and the midpoint of each rear detector array, the carbon events are separated clearly from the hydrogen peak. A cut on the velocity-ratio spectrum at $R_{v}=0.92$ eliminates most of the events from ${ }^{12} \mathrm{C}$, and yields $\eta^{*}=0.046, \epsilon=0.24$, and $\overline{A_{y}}=0.44 \pm 0.02$. Because the time-of-flight resolution of the 12-detector polarimeter ${ }^{4}$ with a $2.0 \mathrm{~m}$ flight path is better than that of a nine-detector polarimeter ${ }^{5}$ with a $1.0 \mathrm{~m}$ mean flight path, a cut at a specified velocity ratio $\left(e . g ., R_{v}=0.92\right)$ will eliminate a larger fraction of events from ${ }^{12} \mathrm{C}$.

\section{Lead-Steel Collimator}

Based on measurements of neutrons in test runs at Bates, ${ }^{6}$ the neutron polarimeter 
Table I. Performance of the polarimeter as a function of the $R_{v}$ cut.

\begin{tabular}{|cccccc|}
\hline$R_{v}$ cut & \% retained & $\bar{\xi} \pm \Delta \bar{\xi}$ & $\overline{A_{y}} \pm \Delta \overline{A_{y}}$ & $\epsilon$ & $\eta^{*}$ \\
\hline 0.88 & 43.2 & $0.259 \pm 0.007$ & $0.39 \pm 0.01_{7}$ & 0.29 & 0.044 \\
0.89 & 40.5 & $0.274 \pm 0.008$ & $0.41 \pm 0.01_{8}$ & 0.27 & 0.046 \\
0.92 & 35.2 & $0.288 \pm 0.009$ & $0.44 \pm 0.02_{0}$ & 0.24 & 0.046 \\
0.94 & 32.3 & $0.297 \pm 0.010$ & $0.45 \pm 0.02_{1}$ & 0.22 & 0.045 \\
0.95 & 31.1 & $0.304 \pm 0.010$ & $0.46 \pm 0.02_{1}$ & 0.21 & 0.045 \\
\hline
\end{tabular}

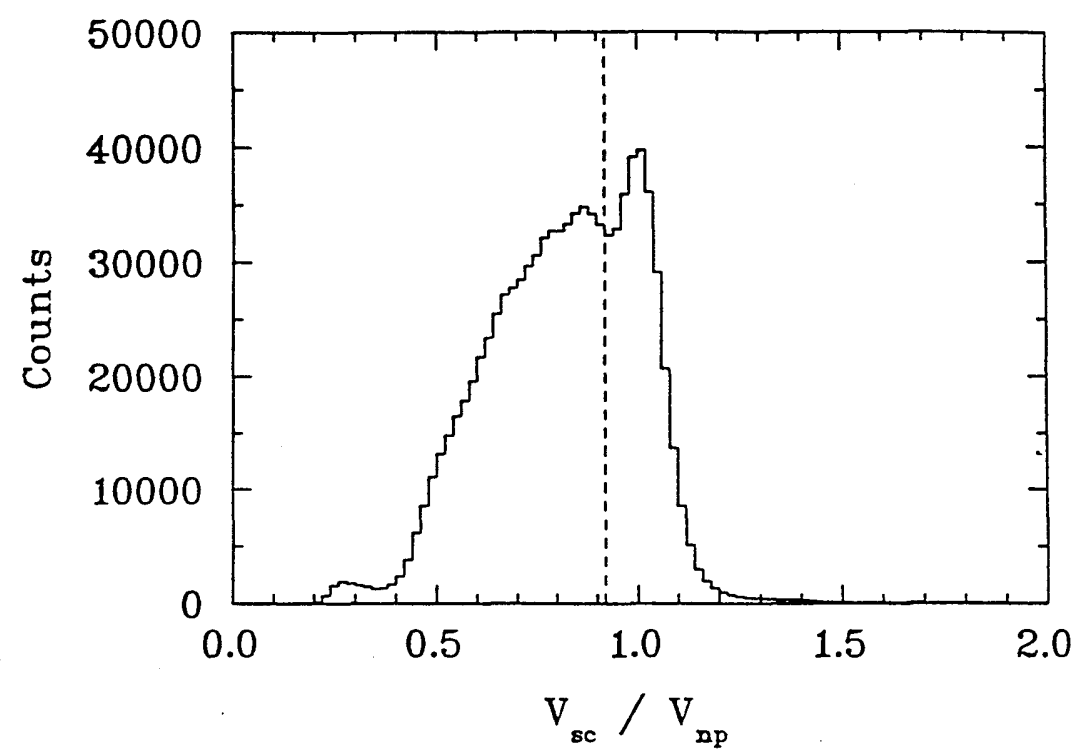

Figure 3. Spectrum of the velocity ratio $R_{\nu}=\nu_{s c} / \nu_{n p}$ for a neutron kinetic energy of $133.8 \mathrm{MeV}$. The dashed line at $R_{\nu}=0.92$ represents a cut that removes most of the events from the inelastic ${ }^{12} C(p, p n)$ reaction.

must be contained in a shielding enclosure with a front wall of several radiation lengths of lead in order to attenuate the energy of high energy photons and the flux of charged particles from the target. To simulate the experimental conditions at Bates, a lead-steel collimator was constructed. The lead-steel collimator consisted of 4 inches of lead sandwiched between two layers of 1.375-inch steel. This sandwiched wall was located ahead of the polarimeter just outside of the hut. The back face of the lead-steel wall was 14.43 inches away from the front face of the first primary scattering scintillator. Ahead of this lead-steel wall was connected a rectangular steel collimator with a cross-sectional opening that measured 20 in high $\times 30$ in wide $\times 48$ in long. The steel that made up this rectangular collimator was 1.375 inches thick. The collimator was surrounded by concrete blocks, 24 in wide $\times 48$ in long. 
The analyzing power $\overline{A_{y}}=0.43 \pm 0.02$ at a velocity ratio cut of 0.92 measured with the lead-steel collimator was the same, within statistics, as that obtained without the collimator. This result is consistent, within statistics, with the prediction that depolarization by the shielding wall should be about $5 \%$; therefore, the use of lead and steel for the shielding in the front wall will not hinder the measurement of $G_{E}^{n}$.

1. R.G. Arnold, C.E. Carlson, and F. Gross, Phys. Rev. C 23, 363 (1981).

2. L. Wolfenstein, Ann. Rev. Nucl. Sci. 6, 43 (1956).

3. G.C. Olsen, Rep. Prog. Phys. 35, 717 (1972).

4. R. Madey, A.R. Baldwin, P.J. Pella, J. Schambach, and R.M. Sellers, IEEE Transactions Nuclear on Science 36, 231 (1989).

5. J.W. Watson, M.R. Plumley, P.J. Pella, B.D. Anderson, A.R. Baldwin, and R. Madey, Nucl. Instr. and Meth. A272, 750 (1988).

6. R. Madey, J. Schambach, B.D. Anderson, A.R. Baldwin, A. Bernstein, G. Dodson, M. Farkhondeh, R. Lourie, J.M. Finn, P. Ulmer, R. Whitney, and J.J. Kelly, in 1987-1988 Annual Scientific and Technical Report, Bates Linear Accelerator Center, p. 140.

\section{FIRST COOLER TEST RUN FOR $\mathrm{pp} \rightarrow \mathrm{pn} \pi^{+}$(CE03)}

W. Daehnick, S. Dytman, W. Brooks, J. Hardie, S. Saha, Hong Li

University of Pittsburgh, Pittsburgh, Pennsylvania 15260

T. Rinckel

Indiana University Cyclotron Facility, Bloomington, Indiana 47405

E.R. Jacobsen

Princeton University, Princeton, New Jersey 08544

On February 20-21, 1990, we made the first Cooler run for this experiment. At that time, an almost full set of CE03 detectors was available. The new, large aperture, $6^{\circ}$ magnet (the central piece of equipment for the T-site experiment) was not installed and the gas jet target was not available, so the anticipated small-angle detection of protons and neutrons from the reaction $\mathrm{pp} \rightarrow \pi$ was not possible at the T-site. However, thin window (127 micron stainless steel) exit ports for charged particles were available on both sides of the beam line covering scattering angles of $38^{\circ}$ to $52^{\circ}$ relative to the beam direction.

A correlated two-arm measurement involving ejectiles of realistic energies was accomplished by using the elastic reaction $\mathrm{pp} \rightarrow \mathrm{pp}$ for incident protons of $150 \mathrm{MeV}$ as a source for simply correlated particles. A $2 \mathrm{~mm}$ thick polyethylene scraper target and a cyclical sweep of the cooled beam into the scraper generated an estimated luminosity of between $10^{26}$ and $10^{27} \mathrm{~cm}^{-2} \mathrm{sec}^{-1}$ at a duty cycle of about $50 \%$.

The "neutron" arm consisted of a 14 element position sensitive scintillator hodoscope (described elsewhere) of area $70 \times 120 \mathrm{~cm}$, with a set of four $3-\mathrm{mm} \Delta \mathrm{E}$ detectors just 